\title{
Correction to: The H741D mutation in Tac1p contributes to the upregulation of CDR1 and CDR2 expression in Candida albicans
}

\author{
Jin-Yan Liu ${ }^{1} \cdot$ Bing Wei $^{2} \cdot$ Ying Wang ${ }^{3} \cdot$ Ce Shi ${ }^{1} \cdot$ Wen-Jing $\mathrm{Li}^{1} \cdot$ Yue Zhao ${ }^{1} \cdot$ Ling-Ning Meng $^{1} \cdot$ Ming-Jie Xiang ${ }^{1}$ \\ Published online: 13 August 2020 \\ (C) Sociedade Brasileira de Microbiologia 2020
}

\section{Correction to: Brazilian Journal of Microbiology}

https://doi.org/10.1007/s42770-020-00336-8

Unfortunately, an error occurred in the author affiliations. The corrected affiliation of Jin-Yan Liu, Ce Shi, Wen-Jing Li, Yue Zhao, Ling-Ning Meng, and Ming-Jie Xiang is given below. Department of Laboratory Medicine, RuiJin Hospital/Lu Wan Branch, School of Medicine, Shanghai Jiaotong University, No. 197 Ruijin Second Road and No. 149 South Chongqing Road, Shanghai, China

Publisher's note Springer Nature remains neutral with regard to jurisdictional claims in published maps and institutional affiliations.

The online version of the original article can be found at https://doi.org/ $10.1007 / \mathrm{s} 42770-020-00336-8$

Ming-Jie Xiang

mjxiang123456@126.com

1 Department of Laboratory Medicine, RuiJin Hospital / Lu Wan Branch, School of Medicine, Shanghai Jiaotong University, No. 197 Ruijin Second Road and No. 149 South Chongqing Road, Shanghai, China

2 Department of Laboratory Medicine, The International Peace Maternity \& Child Health Hospital, Shanghai Jiao Tong University School of Medicine, Shanghai, China

3 Department of Clinical Laboratory, Southern District of Anhui Provincial Hospital, Hefei, China 